\title{
EXPLORANDO CONTEÚDOS MATEMÁTICOS A PARTIR DE TEMAS AMBIENTAIS
}

\author{
Exploring mathematical content \\ through environmental subjects
}

\author{
Maria Beatriz Ferreira Leite ${ }^{1}$ \\ Denise Helena Lombardo Ferreira ${ }^{2}$ \\ Cintia Rigão Scrich ${ }^{3}$
}

\begin{abstract}
Resumo: A Educação Ambiental constitui um processo educativo, cuja finalidade é desenvolver instrumentos pedagógicos e ampliar a prática educativa para que o homem viva em harmonia com o meio ambiente. A Modelagem Matemática é uma estratégia de ensino e aprendizagem, na qual os alunos transformam situações da realidade em modelos matemáticos por meio da investigação, ação e validação, abordando problemas que estão relacionados ao cotidiano. A Modelagem possibilita a aprendizagem de conteúdos matemáticos aplicados em outras ciências, onde a Matemática é aprendida e entendida como um instrumento para a compreensão e possível modificação da realidade. Assim, os modelos matemáticos podem constituir uma ferramenta útil na conscientização do indivíduo enquanto sujeito da sua própria ação. Neste trabalho os temas abordados são: crescimento de peixes, poluição do ar e agrotóxicos.
\end{abstract}

Palavras-chave: Modelagem matemática. Ensino e aprendizagem. Educação ambiental.

\begin{abstract}
Environmental education is an educative process which aims at developing teaching tools and extending educative practice to make human beings live in harmony with the environment. Mathematical Modeling is a strategy for teaching and learning, in which the students change situations of reality into mathematical models through investigation, action and validating, approaching problems which are related to their everyday lives. Modeling enables the learning of mathematical content applied to other sciences, where Mathematics is learned and understood as a tool for the comprehension and possible change of reality. Thus, mathematical models can be a useful tool to make us conscious while subject of our own action. In this work, the themes approached are: fish growth; air pollution and toxic agrochemicals.
\end{abstract}

Keywords: Mathematical modeling. Teaching and learning. Environmental education.

\footnotetext{
${ }^{1}$ Doutora em Matemática Aplicada. Docente da Faculdade de Matemática, CEATEC, Pontifícia Universidade Católica de Campinas (Puccamp). Campinas, SP. < bialeite@puc-campinas.edu.br>

${ }^{2}$ Doutora em Educação Matemática. Docente da Faculdade de Matemática, CEATEC, Puccamp. Campinas, SP.

<lombardo@puc-campinas.edu.br>

${ }^{3}$ Doutora em Engenharia Elétrica. Docente do Instituto de Ensino Superior de Americana (IESA). Americana, SP. <cintia_rs@uol.com.br>

${ }^{1}$ Faculdade de Matemática, CEATEC, Puccamp Rod. D. Pedro I, km 136 - Parque das Universidades Campinas, SP 


\section{Introdução}

A Educação Ambiental constitui um processo educativo, cuja finalidade é desenvolver instrumentos pedagógicos e ampliar a prática educativa para que o homem viva em harmonia com o meio ambiente (BARCELOS, NOAL, 2000; DIAS, 1994).

O capítulo 36 da Agenda $21^{4}$ destaca a Educação Ambiental como um processo que busca desenvolver uma população que seja consciente e preocupada com o meio ambiente e com problemas a ele relacionados; e que tenha conhecimentos, habilidades, atitudes, motivações e compromissos para trabalhar individual e coletivamente, na busca de soluções para os problemas existentes e para a preservação do que ainda resiste e que merece mais atenção.

As linhas gerais de orientações para o desenvolvimento da Educação Ambiental foram discutidas no Encontro de Belgrado (1975), apresentadas na Conferência Intergovernamental sobre Educação Ambiental em Tbilisi (1977), aperfeiçoadas no Congresso Internacional sobre Educação e Formação Ambiental em Moscou (1987) e corroboradas na Rio-92 (Agenda 21) (DIAS, 1994). Da Conferência Rio-92 resultaram os seguintes objetivos:

a)reorientar a educação para o movimento sustentável;

b) aumentar/incrementar a conscientização popular;

c) considerar o analfabetismo ambiental;

d) promover treinamento.

A situação ambiental tem originado preocupações de ordem científica e, ao mesmo tempo, políticas. A mídia tem contribuído para despertar o interesse dos alunos pela temática ambiental, revelando várias questões dessa ordem e fornecendo dados e estimativas. Assim, parece natural utilizar a Matemática, uma disciplina em que a maioria apresenta dificuldades, para analisar e solucionar problemas referentes às questões ambientais. Dessa forma os conteúdos matemáticos surgem naturalmente para que seja possível compreender e analisar tais problemas, podendo levar os alunos a construir hipóteses, pesquisar e ver a Matemática de forma mais contextualizada e significativa.

A junção da Matemática com o meio ambiente deve converter-se num processo criativo que constitui a porta de intercâmbio e fertilização dos comportamentos cotidianos e da consciência dos valores culturais.

A Modelagem Matemática, entendida como uma estratégia de ensino e aprendizagem, na qual os alunos transformam problemas da realidade em problemas matemáticos por meio da investigação, ação e validação, possibilita trazer a realidade para a sala de aula, abordando problemas que estão relacionados ao cotidiano dos alunos, viabilizando a interação da Matemática na sala de aula com aquela existente na realidade.

Ao trabalhar com situações reais, os alunos manipulam dados reais, havendo necessidade de coletar informações e interpretá-las. Como consequência, os alunos caminham para a construção do conhecimento, para o pensamento crítico e reflexivo.

${ }^{4} \mathrm{O}$ documento Agenda 21 resultou da Conferência da ONU, Rio-92. 
Explorando conteúdos matemáticos a partir...

A Modelagem possibilita a aprendizagem dos conteúdos de Matemática conectados a outras ciências, como, por exemplo, a Educação Ambiental, onde a Matemática é aprendida e entendida como um instrumento para a compreensão e possível modificação da realidade.

Espera-se que o envolvimento da Modelagem Matemática com a Educação Ambiental contribua para a formação de um indivíduo ético, criativo e crítico, e que possa viver em uma sociedade de forma participativa e consciente.

A seguir são explorados alguns modelos matemáticos a partir de temas ambientais.

\section{Modelos matemáticos}

Nesta seção pretendemos exemplificar como a matemática pode auxiliar na compreensão, análise e estudo de fenômenos ambientais e como a educação ambiental pode ser útil na exploração de conteúdos matemáticos. Para isso, serão abordados diferentes assuntos, abrangendo conteúdos matemáticos do Ensino Fundamental até o Ensino Superior.

\section{Modelo I: Peixes}

A atividade de pesca amadora no Brasil cresceu consideravelmente nos últimos anos, constituindo não apenas uma atividade de lazer. O Programa Nacional de Desenvolvimento da Pesca Amadora (PNDPA), criado em 1997 e atualmente sob a responsabilidade do IBAMA, destina-se a transformar a atividade de pesca amadora em instrumento de desenvolvimento econômico, social e de conservação ambiental, fortalecendo-a como importante atividade para o turismo, comércio e indústria e, também, para a conservação do meio ambiente (BRASIL, 2007a). Entre outras ações, este projeto orienta e normatiza a pesca amadora, apresentando, por exemplo: tabelas de cotas de captura, tamanho mínimo permitido para captura etc.

O objetivo central deste modelo é justificar matematicamente os comprimentos mínimos permitidos para captura, apresentados pelo IBAMA, com base nos dados obtidos nas campanhas de pesca do Grupo de Pesquisa em Ecossistemas Aquáticos sujeitos a Impactos Ambientais da PUC-Campinas, coletados no período de 2005 a 2006 na Represa de Jaguari (Sistema Cantareira - SABESP). Estão relacionados, na Tabela 1, os valores médios obtidos para comprimento e peso para a espécie Schizodon nasutus, conhecida popularmente como taguara. Sabe-se, também (FROESE, PAULY, 2007), que o comprimento máximo deste peixe é de $33,7 \mathrm{~cm}$.

\begin{tabular}{cc}
\multicolumn{2}{l}{$\begin{array}{l}\text { Tabela 1. Valores médios para } \\
\text { comprimento e peso do Schizodon nasutus }\end{array}$} \\
\hline Peso (gramas) & Comprimento (cm) \\
\hline 23,7 & 12,5 \\
54,5 & 17,5 \\
113 & 22,5 \\
\hline
\end{tabular}


Leite, M. B. F.; Ferreira, D. H. L.; Scrich, C. R.

Buscando estabelecer uma relação entre comprimento e peso, com base nos dados da Tabela 1, será utilizado o princípio da alometria, frequentemente usado em Biomatemática. Este princípio estabelece que, num mesmo indivíduo, "a razão entre os crescimentos específicos (relativos) de seus órgãos é constante". Sendo $\mathrm{P}(\mathrm{t})$ e $\mathrm{C}(\mathrm{t})$ as respectivas medidas para peso e comprimento dos órgãos ou partes distintas do corpo de um mesmo peixe no instante t, a relação alométrica traduz-se pela seguinte equação diferencial:

$$
\frac{1}{\mathrm{P}} \frac{d \mathrm{P}}{d t}=\alpha \frac{1}{\mathrm{C}} \frac{d \mathrm{C}}{d t}
$$

onde $P(t)>0, C(t)>0$, e $\alpha$ é chamado coeficiente de alometria. De (1) obtemos a solução $P=a C^{\alpha}$. Os valores dos parâmetros a e $\alpha$ foram estimados a partir da linha de tendência obtida através do EXCEL, resultando na seguinte função representada na Figura 1.

O PNDPA (BRASIL, 2007a) estabelece que o comprimento mínimo permitido para captura desta espécie é $25 \mathrm{~cm}$, isto é, se um peixe tiver menos de $25 \mathrm{~cm}$ de comprimento, deve ser devolvido ao seu ambiente. Neste sentido, supomos que este valor relaciona-se com a preservação da espécie e, portanto, destina-se a garantir que o peixe não seja capturado antes da reprodução. Além disso, para algumas espécies, o amadurecimento das gônadas (glândulas sexuais), condição necessária para o acasalamento, ocorre quando a variação do crescimento é máxima (BASSANEZI, 2002).

É possível descrever crescimento populacional (neste caso, o peso) em função do tempo por intermédio de vários modelos matemáticos. Dentre os modelos que pressupõem um crescimento limitado, um dos mais simples é o modelo logístico, que incorpora um fator

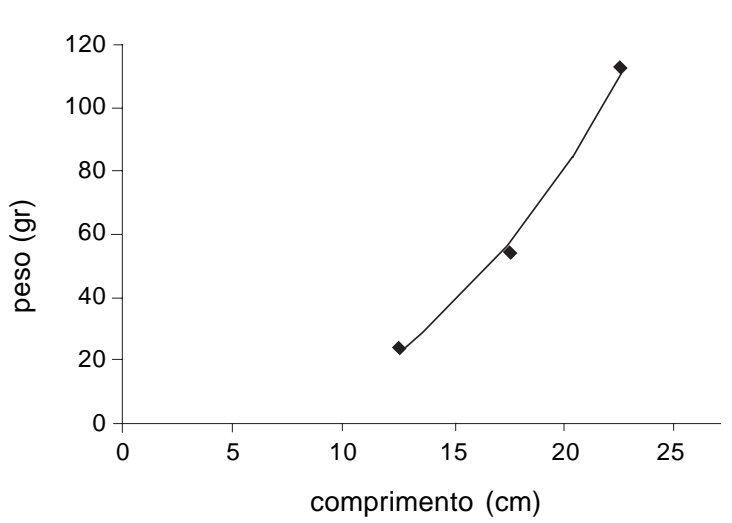

Figura 1. Relação alométrica para Schizodon nasutus. 
Explorando conteúdos matemáticos a partir...

inibidor, fazendo com que o peso se estabilize num valor máximo (BASSANEZI, 2002). A equação diferencial que descreve este modelo é

$$
\frac{d \mathrm{P}}{d t}={ }^{r} P\left(1-\frac{P}{\mathrm{P}_{\text {máx }}}\right)
$$

com $r>0$ e $\mathrm{P}_{\text {máx }}$ é o peso máximo atingido.

A estimativa de $\mathrm{P}_{\text {máx }}$ pode ser obtida através da relação alométrica $\mathrm{P}=0,0291 \mathrm{C}^{2,6473}$ substituindo-se o comprimento máximo de $33,7 \mathrm{~cm}$. Assim, estimamos $\mathrm{P}_{\text {máx }}=322 \mathrm{~g}$.

Uma análise matemática do modelo mostra que a maior variação de crescimento $\frac{\mathrm{d}^{2} \mathrm{P}}{\mathrm{dt}^{2}}=0$ ocorre quando $\mathrm{P}_{=}=\frac{\mathrm{P}_{\text {máx }}}{2}=161$ gramas.

Substituindo esse valor na relação alométrica, obtemos $C=25,93 \mathrm{~cm}$, o que justifica o valor apresentado pelo IBAMA, apesar do pequeno número de dados disponíveis na Tabela 1.

Outra questão pertinente é: por que não considerar diretamente a variação temporal do comprimento? Isto seria possível se o modelo escolhido fosse outro (por exemplo, Montroll (BASSANEZI, 2002)) pois, no modelo logístico, a variação máxima do crescimento sempre ocorre em $\mathrm{P}_{\text {max }} / 2$, e se a variável fosse o comprimento, teríamos que a maturação das gônadas ocorreria quando o peixe atinge $16,85 \mathrm{~cm}$, o que não valida a informação do IBAMA.

Nível: Ensino Superior

Conteúdos matemáticos explorados: Funções, Limites, Derivadas e suas aplicações, Mínimos Quadrados, dentre outros.

\section{Modelo 2: Poluição do ar}

A poluição atmosférica, mesmo com níveis abaixo do permitido pelos órgãos responsáveis, afeta significativamente a vida dos seres vivos (MARTINS et al., 2002).

As faixas etárias mais sensíveis aos efeitos da poluição atmosférica são as crianças e os idosos, apresentando maior suscetibilidade a doenças respiratórias causadas ou agravadas por poluentes atmosféricos. Como meio de diminuir os níveis de poluição atmosférica, alguns países adotam o sistema de rodízio de veículos, visto que essa é uma das principais fontes poluidoras.

$\mathrm{Na}$ cidade de Campinas, a concentração de poluentes é medida diariamente, e está disponível nos relatórios da CETESB (BRASIL, 2007b). Para exemplificar, são utilizadas as concentrações do poluente MP10, que são pequenas partículas inaláveis, com diâmetro aerodinâmico menor ou igual a 10 micrômetros, penetrando com maior facilidade nas vias respiratórias (www.metrologiaquimica.com.br). Este poluente foi considerado por estar diretamente relacionado com a poluição atmosférica causada pelos veículos automotores.

Com base nos dados disponibilizados pela CETESB (BRASIL, 2007b), foram calculadas as médias mensais de janeiro a dezembro de 2006, resultando na Tabela 2. 
Leite, M. B. F.; Ferreira, D. H. L.; Scrich, C. R.

Tabela 2. Médias mensais de PM10 na cidade de Campinas.

\begin{tabular}{ccccccccccccc}
\hline Mês & $\mathbf{1}$ & $\mathbf{2}$ & $\mathbf{3}$ & $\mathbf{4}$ & $\mathbf{5}$ & $\mathbf{6}$ & $\mathbf{7}$ & $\mathbf{8}$ & $\mathbf{9}$ & $\mathbf{1 0}$ & $\mathbf{1 1}$ & $\mathbf{1 2}$ \\
\hline PM10 & 22,7 & 22,5 & 32,5 & 35,1 & 44,6 & 46,2 & 48,9 & 48 & 45,1 & 36,9 & 33,6 & 26,8 \\
\hline
\end{tabular}

A representação dos valores da Tabela 2 sugere uma variação sazonal que pode ser aproximada por uma função trigonométrica. Escolhendo-se, por exemplo, uma função seno, explora-se a forma genérica,

$$
\mathrm{y}=\mathrm{A}+\mathrm{B} \operatorname{sen}\left(\frac{2 \pi}{D}(t+C)\right)
$$

onde A é o deslocamento vertical, B é a amplitude, C é o deslocamento horizontal e D é o período.

A estimativa para esses parâmetros foi feita por meio de sucessivas tentativas e observações do gráfico resultante. Não foi utilizada nenhuma ferramenta matemática para aproximação e/ou ajuste de funções, pois são enfocados conteúdos contemplados no Ensino Médio. Dentre as diversas tentativas, a função escolhida foi

$$
y=34+10 \operatorname{sen}\left(\frac{2 \pi}{12}(t-4)\right)
$$

representada na Figura 2. A representação gráfica evidencia o aumento do nível do poluente no período de maio a agosto, o que coincide com o período no qual a ocorrência de doenças respiratórias é maior e a umidade do ar mais baixa. Deste modo, o modelo apresentado poderia ser utilizado, por exemplo, para justificar a adoção do rodízio de veículos nos períodos onde a concentração de poluentes é mais alta.

Os dados apresentados possibilitam a exploração de outras funções trigonométricas, como a função cosseno. Diferentes enfoques relacionados à poluição do ar permitem a construção de modelos matemáticos que abrangem o estudo de outras funções, dependendo da natureza dos dados obtidos (JORGE, LEITE, 2006).

Nível: Ensino Médio

Conteúdos matemáticos explorados: média aritmética, gráficos, função trigonométrica, dentre outros. 
Explorando conteúdos matemáticos a partir...

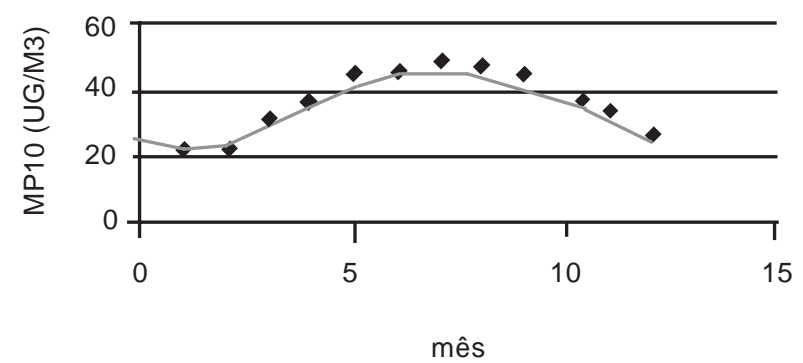

Figura 2. Quantidade média mensal de MP10 na cidade.

\section{Modelo 3: Alimentos orgânicos}

A preocupação com o consumo de alimentos que não contêm agrotóxicos vem aumentando a cada dia. A utilização dos agrotóxicos, além de arriscar a vida de trabalhadores rurais (não são raros os casos de intoxicação por agrotóxicos), compromete a qualidade dos alimentos e, também, contamina o solo e a água. Uma alternativa para se evitar danos ambientais com a utilização de agrotóxicos, fertilizantes e outros produtos químicos é a agricultura orgânica, que pode ser definida como um sistema integrado de manejo, que promove a manutenção da agrobiodiversidade e dos ciclos biológicos, buscando a sustentabilidade social, ambiental e econômica da unidade de produção. O princípio básico desse sistema é a conservação dos recursos naturais.

Como exemplo, pode-se considerar a produção de tomate, visto que é um alimento que, quando cultivado com agrotóxicos, apresenta altos índices de toxinas.

Além dos benefícios ambientais, os produtos orgânicos possuem, em geral, um valor nutritivo maior, quando comparados com os produtos convencionais. Borguini (2002) apresenta a composição do tomate Carmem (Tabela 3).

Considerando-se que, para uma dieta balanceada, entre outros nutrientes, um indivíduo necessita ingerir diariamente $0,01 \mathrm{~g}$ (por kg) de cálcio; $0,02 \mathrm{~g}$ (por $\mathrm{kg}$ ) de fósforo; $75 \mathrm{mg}$ de vitamina C (TORRES, 1992), e supondo, por exemplo, que 5\% dessas necessidades sejam supridas por meio do consumo de tomate Carmem, o modelo proposto pretende estabelecer uma comparação entre as quantidades consumidas dos dois tipos de tomate Carmem, orgânico e convencional.

Por exemplo, um indivíduo de $60 \mathrm{~kg}$, precisa ingerir, diariamente, por meio do consumo de tomate Carmem, 0,03g de cálcio, $0,06 \mathrm{~g}$ de fósforo e 3,75 $\mathrm{mg}$ de vitamina C. A Tabela 4 ilustra as quantidades de cada tipo de tomate para que estas necessidades sejam atendidas, cujos cálculos basearam-se nas informações da Tabela 3 . 
Leite, M. B. F.; Ferreira, D. H. L.; Scrich, C. R.

Observa-se, na Tabela 4, que, em relação à quantidade de fósforo, o tomate Carmem orgânico é mais vantajoso em relação ao convencional; já em relação à quantidade de cálcio, $\mathrm{O}$ convencional supera o orgânico. Desta forma, perguntamos como, a partir da combinação dos dois tipos (orgânico e convencional), podemos obter 5\% da quantidade diária necessária dos nutrientes para uma pessoa de sessenta quilos. Com a intenção de elaborar um modelo matemático que aborde conteúdos matemáticos do Ensino Fundamental, os sistemas lineares propostos consideram apenas dois tipos de nutrientes simultaneamente. Salientamos que, se o objetivo fosse elaborar modelos para o Ensino Médio ou Ensino Superior, os sistemas poderiam acoplar os três tipos de nutrientes simultaneamente.

Tabela 3. Composição do tomate orgânico e convencional.

\begin{tabular}{lcc}
\hline & Produção convencional & Produção orgânica \\
\hline Água (\%) & 94,53 & 94,53 \\
Nitrogênio (mg/100g) & 114,87 & 136,31 \\
Fósforo (mg/100g) & 20,24 & 23,36 \\
Potássio (mg/100g) & 129,75 & 146,49 \\
Cálcio (mg/100g) & 9,03 & 6,02 \\
Magnésio (mg/100g) & 7,06 & 8,53 \\
Enxofre (mg/100g) & 7,17 & 10,28 \\
Vitamina C (mg/100g) & 21,90 & 22,90 \\
\hline
\end{tabular}

Fonte: <www.tese.usp.br/teses/disponíveis>

Tabela 4. Quantidade do tomate Carmem (g) para suprir 5\% das necessidades diárias de uma pessoa de sessenta quilos.

\begin{tabular}{lcc}
\hline & Tomate convencional & Tomate orgânico \\
\hline Fósforo & & \\
Cálcio & 296,4 & 256,85 \\
Vitamina C & 332,23 & 498,34 \\
& 17,12 & 16,37 \\
\hline
\end{tabular}

Sendo x a quantidade (em $100 \mathrm{~g}$ ) do tomate Carmem convencional e y a quantidade (em $100 \mathrm{~g}$ ) do tomate Carmem orgânico, as equações que descrevem as quantidades desejadas são dadas pelos sistemas lineares a seguir. Observamos que, como os dados da Tabela 3 estão em miligramas, as quantidades necessárias dos nutrientes também são expressas nesta unidade.

$$
\begin{aligned}
& \text { (I) }\left\{\begin{array}{cl}
20,24 x+23,36 y=60 & \text { (fósforo) } \\
9,03 x+6,02 y=30 & \text { (cálcio) }
\end{array}\right. \\
& \text { (II) }\left\{\begin{array}{cl}
20,24 x+23,36 y=60 & \text { (fósforo) } \\
21,9 x+22,9 y=3,75 & \text { (vitamina C) }
\end{array}\right. \\
& \text { (III) }\left\{\begin{array}{cl}
9,03 x+6,02 y=30 & \text { (cálcio) } \\
21,9 x+22,9 y=3,75 & \text { (vitamina C) }
\end{array}\right.
\end{aligned}
$$


Explorando conteúdos matemáticos a partir...

As soluções obtidas algebricamente para os sistemas I, II e III são, respectivamente $(3,8 ;-0,7),(-26,7 ; 25,7)$ e $(8,9 ;-8,3)$, que não fazem sentido, neste caso, por apresentarem valores negativos. Para explorar essa situação, podemos representar graficamente o sistema e analisar a região de viabilidade para o problema. A Figura 3 representa o sistema I. Para os outros sistemas, a análise é similar. A região hachurada é a região viável para se encontrarem as soluções do sistema. Observe que a solução encontra-se fora da mesma, indicando que, na região viável, a reta que representa a necessidade de fósforo está sempre abaixo daquela que determina a quantidade de cálcio.

Salientamos ainda que, incorporando ao sistema informações relativas a outros nutrientes, e vinculando essas informações com o objetivo de, por exemplo, gastar o menor valor possível na compra de tomates, é possível abordar outros conteúdos matemáticos, como Programação Linear (Ensino Superior).

Nível: Ensino Fundamental

Conteúdos matemáticos: Conversão de unidades de medidas, regra de três simples, função de primeiro grau, sistemas lineares, gráficos, dentre outros.

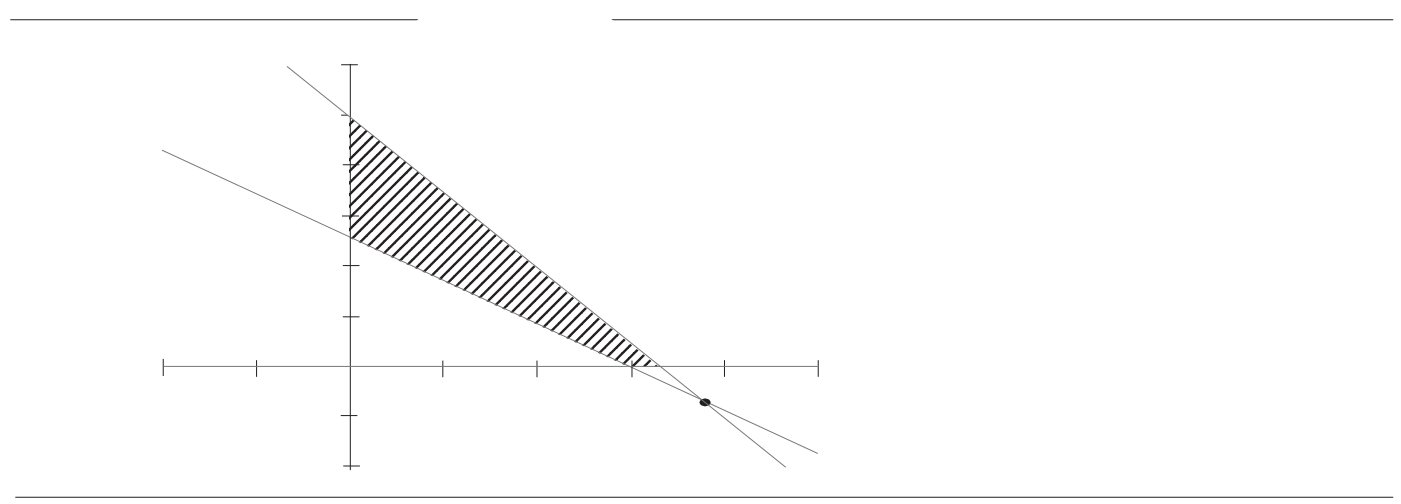

Figura 3. Região viável para as soluções do sistema I.

\section{Considerações finais}

A investigação de temas ambientais possibilitou a exploração de diversos conteúdos matemáticos, como, por exemplo: médias, funções, derivadas, equações diferenciais, sistemas lineares. Por intermédio dos modelos formulados, observamos que o conteúdo matemático abordado depende, basicamente, do enfoque dado e do problema investigado. Entretanto, o mesmo problema pode ser tratado matematicamente de diferentes maneiras, cabendo, ao professor, encaminhar a formulação dos modelos considerando o nível de ensino no qual se deseja trabalhar, atentando para a questão dos pré-requisitos necessários para que o aluno, de fato, consiga usar a matemática de forma significativa e contextualizada.

É importante observar também que todos os modelos matemáticos podem ser melhorados e/ou modificados a partir da formulação de novas hipóteses. Essa percepção de que 
Leite, M. B. F.; Ferreira, D. H. L.; Scrich, C. R.

a matemática não está pronta e acabada também é valiosa para a formação de um indivíduo crítico, incentivando sua criatividade e autonomia. De acordo com Bassanezi (2002), um bom modelo é aquele que propicia a formulação de novos modelos.

A escolha de temas ambientais ocorreu, sobretudo, pela sua importância e necessidade da conscientização sobre os problemas ambientais e suas consequências. Essa preocupação também é destacada por Skovsmose (2001), ao afirmar que é importante a formação de alunos com poder de argumentação por meio do pensamento reflexivo e crítico, com comprometimento com o ambiente em que participa.

\section{Referências}

BARCELOS, V. H. L.; NOAL, F. O. A temática ambiental e a educação: uma aproximação necessária. 2. ed. In: NOAL, F. O.; REIGOTA, M.; BARCELOS, V. H. L. (Orgs.).

Tendências da Educação Ambiental Brasileira. Rio Grande do Sul: Edunisc, 2000. p. 99-114.

BASSANEZI, R. C. Ensino-aprendizagem com modelagem matemática. São Paulo: Contexto, 2002.

BORGUINI, R. G. Tomate (Lycopersicum esculentum mill) orgânico: o conteúdo nutricional e a opinião do consumidor. 2002. 110f. Dissertação (Mestrado) - Escola Superior de Agricultura Luiz de Queiroz, Universidade Estadual de São Paulo, Piracicaba, 2002.

BRASIL. Ministério do Meio Ambiente. Programa Nacional de Desenvolvimento da Pesca Amadora PNDPA. Disponível em: <http://www.ibama.gov.br/pndpa>. Acesso em: 12 fev. 2007a.

. Portal do Governo do Estado de São Paulo. Disponível em: <http://

www.cetesb.sp.gov.br/ar>. Acesso em: 23 fev. 2007b.

DIAS, G. F. Atividades Interdisciplinares de Educação Ambiental: manual do professor. São Paulo: Global/Gaia, 1994.

FROESE, R.; PAULY, D. (Eds.). FishBase. Disponível em: <www.fishbase.org>. Acesso em: 25 jan. 2007.

JORGE, F. C. R.; LEITE, M. B. F. Modelos matemáticos e temas interdisciplinares. In: ENCONTRO DE INICIAÇÃO CIENTÍFICA, 11., 2006, Campinas. Resumos...

Campinas: Puccamp, 2006. p. 62.

MARTINS, L. C. et al. Poluição atmosférica e atendimentos por pneumonia e gripe em São Paulo, Brasil. Revista de Saúde Pública, São Paulo, v. 36, n. 1, p. 88-94, 2002.

SKOVSMOSE, O. Educação matemática crítica: a questão da democracia. Campinas: Papirus, 2001.

TORRES, J. D. Cozinhe sem carne. São Paulo: Cone, 1992.

Artigo recebido em julho de 2008 e aceito em fevereiro de 2009 